\title{
Effect of correlations and disorder on electron states in the Mott-Hubbard insulator $\mathrm{V}_{2} \mathrm{O}_{3}$
}

\author{
S. A. Carter \\ The James Franck Institute and Department of Chemistry, The University of Chicago, Chicago, Illinois 60637 \\ J. Yang and T. F. Rosenbaum \\ The James Franck Institute and Department of Physics, The University of Chicago, Chicago, Illinois 60637 \\ J. Spalek \\ Department of Physics, Purdue University, West Lafayette, Indiana 47907 \\ J. M. Honig \\ Department of Chemistry, Purdue University, West Lafayette, Indiana 47907 \\ (Received 25 June 1990)
}

\begin{abstract}
We compare vanadium-deficient (nonstoichiometric) and titanium-doped vanadium sesquioxide through measurements of the electrical resistivity at a series of hydrostatic pressures, the magnetic susceptibility, and the low-temperature specific heat: all as a function of $T$. The pressure dependence of the critical temperature for the discontinuous metal-antiferromagnetic-insulator transition as well as the temperature dependence of the magnetic susceptibility track in the two cases. However, the pressure dependence of the Hubbard gap, the slower than exponential form of the lowtemperature resistivity, and the concentration of two-level systems are markedly different for $\mathrm{V}_{1.9967} \mathrm{O}_{3}$ and $\left(\mathrm{V}_{0.99} \mathrm{Ti}_{0.01}\right)_{2} \mathrm{O}_{3}$. We discuss our results in terms of the intra-atomic Coulomb repulsion, which is of comparable magnitude to the bare bandwidth of the vanadium $3 d$ states. The band splitting in the antiferromagnetic insulating state is argued to cross over to a Slater-type splitting between the subbands narrowed by correlations with a sufficient degree of oxygen nonstoichiometry or Ti doping.
\end{abstract}

\section{INTRODUCTION}

Pure vanadium sesquioxide undergoes a striking firstorder metal-insulator transition at a temperature $T_{M I} \sim 150 \mathrm{~K}$, marked by a jump in the electrical resistivity of seven orders of magnitude, a hysteresis loop of 10 to $12 \mathrm{~K}$, a volume expansion of $1.4 \%$, and antiferromagnetic ordering of the spins. Investigations ${ }^{1}$ have centered on the remarkable electronic, magnetic, and structural behavior at the transition, which has served as a prototype for the physics of Mott-Hubbard transitions. Moreover, the metallic phase can be stabilized at all $T$ through alloying with titanium sesquioxide, adjustment of the oxygen stoichiometry, and/or the application of sufficient hydrostatic pressure. These additional experimental parameters allow both the study of the evolution of the nature of the transition into the insulator and the characterization of the low-temperature correlated metal. ${ }^{2,3}$ The properties of $\mathrm{V}_{2} \mathrm{O}_{3}$ and its alloys are of particular interest in light of the discovery of superconductivity in the rareearth cuprate oxides, where doping transforms a putative Mott-Hubbard insulator into a high-temperature superconductor. The vanadium sesquioxide systems also can be regarded as prototypes of a heavy fermion metal composed of almost localized electrons.

We report here a study of the low-temperature properties of the insulating state of both Ti-doped and vanadium-deficient single crystals of $\mathrm{V}_{2} \mathrm{O}_{3}$ in an attempt to understand the relative roles of disorder and Coulomb repulsion. The latter can be characterized by the ratio $U / W$, where $U$ is the magnitude of the intra-atomic interaction and $W$ is the bare bandwidth. By use of a hydrostatic pressure cell, we can effectively vary $W$ while maintaining the integrity of the samples and contacts through the metal-insulator transition. Thus, we are able to extend electrical resistivity measurements to temperatures well below $T_{M I}$. We supplement the transport measurements with both ac magnetic susceptibility and calorimetric comparisons of the nonstoichiometric and substitutionally disordered insulators. We interpret our findings within the Hubbard-Gutzwiller picture of correlated electron states, additionally taking into account the disorder introduced in the hole states by vanadium deficiency or Ti doping.

\section{EXPERIMENTAL METHODS}

Single crystals of Ti-doped $\mathrm{V}_{2} \mathrm{O}_{3}$ were grown from the melt by the Reed Tri-arc technique. ${ }^{4}$ Nonstoichiometric single crystals were grown using a skull melter. ${ }^{5}$ In both cases, samples were annealed in a suitable $\mathrm{CO}-\mathrm{CO}_{2}$ atmosphere for control of stoichiometry, ${ }^{6}$ quenched, aligned, and cut on a diamond saw. Surfaces were sanded and etched in $\mathrm{HCl}$ prior to attaching electrical leads with silver paint. 
We made four-probe resistivity measurements, $\rho(T)$, on oriented samples of typical dimension $1 \times 1 \times 3 \mathrm{~mm}^{3}$ in a $\mathrm{BeCu}$ hydrostatic pressure cell, using silicone oil as the pressure medium and a superconducting lead manometer for pressure $P$ calibration. We estimate an absolute accuracy of $0.5 \mathrm{kbar}$ for the reported values of $P$. Pressure helped maintain the electrical contacts through the $1.4 \%$ volume expansion at the metal-insulator transition, although the $\mathrm{V}_{1.9967} \mathrm{O}_{3}$ results were quite noisy at low $T$. Transport properties were measured using an ac resistance bridge technique at lowest $\rho$, a four-probe dc technique at intermediate $\rho$, and a Keithley 617 electrometer up to $10^{11} \Omega$, with overlap of at least an order of magnitude between techniques. All results were obtained in the ohmic limit.

We measured the ac magnetic susceptibility at ambient pressure using a gradiometer configuration, with 43 turns/cm of 36-gauge $\mathrm{Cu}$ wire in the primary and 1000 turns of 36-gauge $\mathrm{Cu}$ wire in each of the secondaries. Samples of typical dimension $3 \times 3 \times 10 \mathrm{~mm}^{3}$ aligned along either the $a$ or $c$ axis of the hexagonal unit cell were encapsulated in a quartz tube, which was lifted in and out of the coils at each temperature to subtract the remaining background signal. Measuring fields were restricted to less than $3 \mathrm{G}$, and all results were reported in the magnetic field and for the frequency-independent regime.

We studied the low-temperature heat capacity in a helium dilution refrigerator using a standard relaxation technique. Samples of mass 0.22 and $0.58 \mathrm{~g}$ for the Tidoped and vanadium-deficient samples, respectively, were ground up and mixed with Apiezon $\mathbf{N}$ grease to minimize problems with cracking at the transition. The slurry was spread on a quartz substrate with a gold heater evaporated on the back and suspended by threads from the mixing chamber. The temperature was measured using a Spear carbon chip, calibrated against a germanium thermometer. We estimate a negligible addendum at all $T$, except for the contribution from the Apiezon $N$ grease which was subtracted using known values in the literature. Temperature excursions never exceeded $4 \%$ of the measuring $T$ above $1 \mathrm{~K}$ and $6 \%$ below $1 \mathrm{~K}$.

\section{RESULTS}

We plot in Fig. 1 the temperature dependence of the $c$ axis electrical resistivity at a series of hydrostatic pressures for both vanadium-deficient and titanium-doped $\mathrm{V}_{2} \mathrm{O}_{3}$. Metallic behavior at high $T$ is abruptly interrupted by a jump in $\rho$ of order four decades for $\left(\mathrm{V}_{0.99} \mathrm{Ti}_{0.01}\right)_{2} \mathrm{O}_{3}$ and up to six decades for $\mathrm{V}_{1.9967} \mathrm{O}_{3}$, marking the transition into the insulator. All curves shown are for the cooling cycle only. Significant hysteresis loops occur between cooling and warming, as discussed below in conjunction with the magnetic susceptibility data.

The metal-insulator transition temperature $T_{M I}$ is reduced with pressure almost identically in the two cases, lending credence to the practice of mapping doped and compressed $\mathrm{V}_{2} \mathrm{O}_{3}$ onto the same phase diagram. ${ }^{8}$ Given that $\mathrm{V}_{1.9967} \mathrm{O}_{3} \equiv \mathrm{V}_{2} \mathrm{O}_{3.005}$, an oxygen excess of 0.005 and a Ti substitution of 0.01 achieve precisely the same reduc-

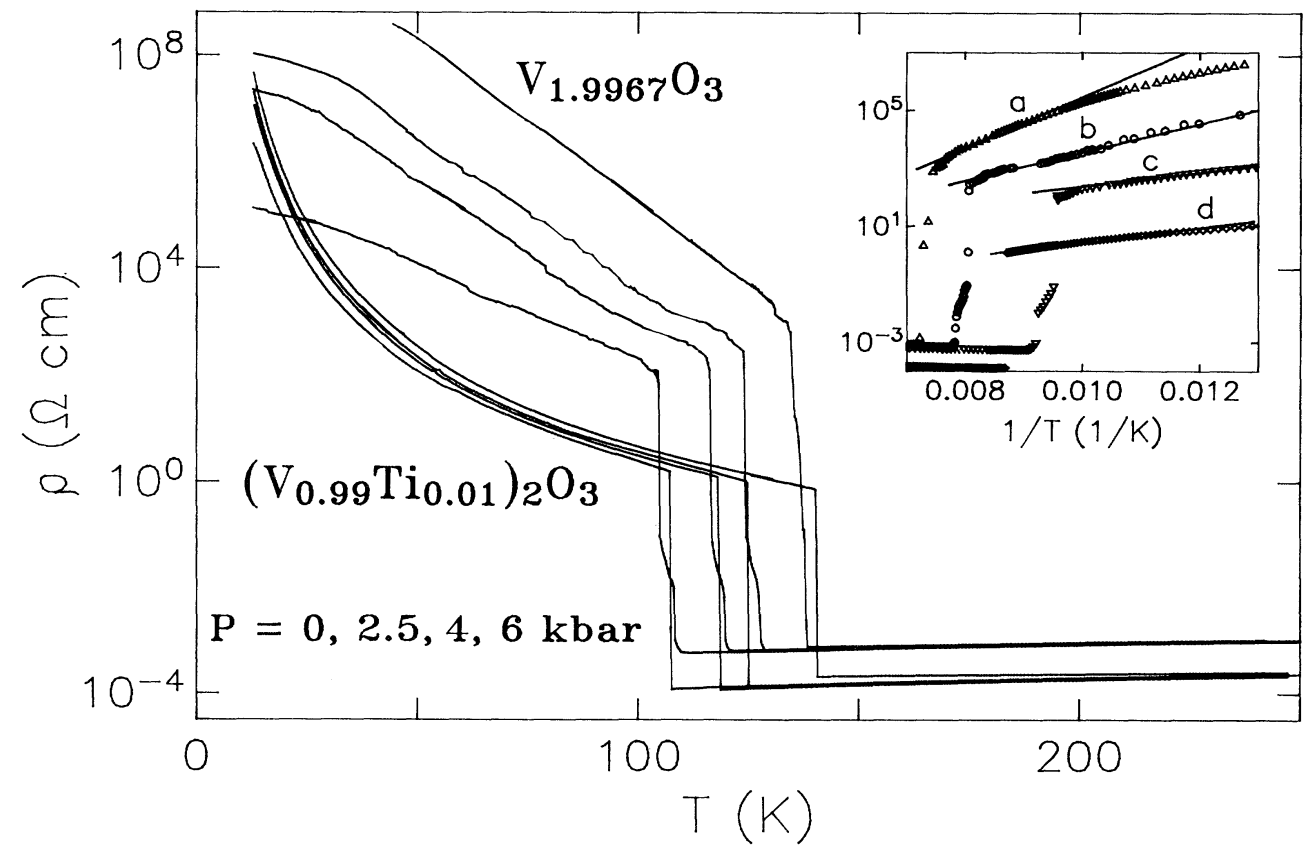

FIG. 1. Resistivity $\rho$ as a function of temperature $T$ at a series of hydrostatic pressures $P$ on cooling. The metal-insulator transition temperature $T_{M I}$ decreases in a like manner with $P$ in the titanium-doped and vanadium-deficient cases, but $\rho(T)$ at low $T$ differs markedly. Inset shows vanadium-deficient data for $P=0,2.5$, and $6 \mathrm{kbar}$ (curves $a-c$ ) and Ti-doped results for $P=4 \mathrm{kbar}$ (curve $d$ ), all vs $1 / T$. Solid lines are best fits to $\rho \propto \exp (\Delta / T)$ for $T \sim 20$ to $30 \mathrm{~K}$ below $T_{M I}$. 
tion in $T_{M I}$. This suggests the applicability of a simple concept in which the oxygen nonstoichiometry creates twice as many holes as the $\mathrm{Ti}$ doping. Thus, the transition seems to be dominated by charge-density effects, and the application of pressure has the same effect on both specimens. However, the nature of the insulating state at equivalent points on the phase diagram appears to be very different, as reflected in the marked contrast between the $\rho(T)$ variation of the two samples for $T<T_{M I}$.

A stretched exponential form for the resistivity,

$$
\rho(T)=\rho_{0} \exp \left(T_{0} / T\right)^{\beta},
$$

with $0<\beta<1$, is a characteristic procedure for dealing with the effects of disorder on the insulating state in the low- $T$ limit. Mott variable range hopping ${ }^{9}$ corresponds to $\beta=1 /(d+1)$, where $d$ is the system dimensionality, whereas $\beta=\frac{1}{2}$ (for both two and three dimensions) when long-range electron-electron interactions in the presence of disorder are sufficiently strong to open a Coulomb gap. ${ }^{10}$ We can fit the temperature evolution of the resistivity for $\left(\mathrm{V}_{0.99} \mathrm{Ti}_{0.01}\right)_{2} \mathrm{O}_{3}$ to Eq. (1) over the entire range $T<T_{M I}$ at all $P$, with $\beta=0.31 \pm 0.02$. However, the prefactor $\rho_{0} \sim 10^{-18} \Omega \mathrm{cm}$ represents an unphysically small value. Moreover, there is no reason to believe that the sample is effectively two-dimensional as implied by the value $\beta=\frac{1}{3}$; in fact, $\rho(T)$ measured along the $a$ axis and the $c$ axis yield virtually identical results.

In light of these considerations, we do not fit $\rho\left(T<T_{M I}\right)$ to a single form, but proceed as follows: First, we fit the region within 20 to $30 \mathrm{~K}$ of $T_{M I}$ to a simple activated form, $\rho(T)=\rho_{1} \exp (\Delta / T)$, representing the opening of a combined Slater-Hubbard gap. We plot in the inset to Fig. $1 \ln \rho$ versus $1 / T$ for $T$ in the near vicinity of $T_{M I}$. Curves $a-c$ represent data for $\mathrm{V}_{1.9967} \mathrm{O}_{3}$ at $P=0,2.5$, and $6 \mathrm{kbar}$, while curve $d$ is for $\left(\mathrm{V}_{0.99} \mathrm{Ti}_{0.01}\right)_{2} \mathrm{O}_{3}$ at $P=4 \mathrm{kbar}$. The slope (solid lines) determines the value of the conductivity gap $\Delta$. Second, we restrict Eq. (1) to the low-temperature limit of our data, $15 \leq T \leq 80 \mathrm{~K}$. Over this temperature range, $\beta=0.26 \pm 0.02$, consistent with three-dimensional Mott variable range hopping, with $\rho_{0} \sim 5 \times 10^{-9} \Omega \mathrm{cm}$, a value which compares well to the prefactors obtained in similar analyses of other vanadium oxides. ${ }^{11}$ The prefactor for the activated behavior, $\rho_{1} \sim 3 \times 10^{-3} \Omega \mathrm{cm}$, which, as expected, is a characteristic resistivity of order the inverse of Mott's minimum metallic conductivity. ${ }^{9}$

The resistivity data for $\mathrm{V}_{1.9967} \mathrm{O}_{3}$ are not amenable to fitting by Eq. (1) over any reasonable temperature range at any pressure. We are able to fit $\rho(T)$ in the 20- to $30-\mathrm{K}$ region just below $T_{M I}$ to a simple activated form, and we plot in Fig. 2 the values of the band gap $\Delta$ so obtained as a function of hydrostatic pressure for both the nonstoichiometric and Ti-doped samples. The gap closes rapidly with increasing $P$ for $\mathrm{V}_{1.9967} \mathrm{O}_{3}$, but remains constant, within experimental error for $\left(\mathrm{V}_{0.99} \mathrm{Ti}_{0.01}\right)_{2} \mathrm{O}_{3}$, at a value which is roughly $\frac{1}{6}$ of the low-temperature gap ${ }^{12}$ in pure $\mathrm{V}_{2} \mathrm{O}_{3}$. At the higher pressures, $\Delta$ is comparable in the two cases, as illustrated by the parallel slopes of curves $c$ and $d$ in the inset to Fig. 1. Increased external pressure seems to drive the two disordered systems to-

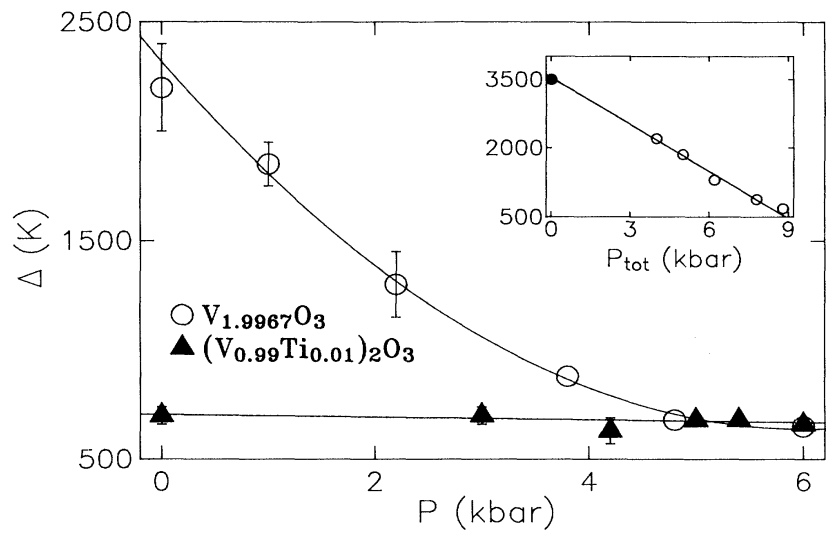

FIG. 2. Variation of the activation energy $\Delta$ with pressure $P$ as deduced from $\rho(T)$. Lines are only guides to the eye. Inset shows $\Delta$ vs the sum of the external and internal (chemical) pressures $P_{\text {tot }}$. Solid line is a linear extrapolation to data for pure $\mathrm{V}_{2} \mathrm{O}_{3}$ (solid circle, Ref. 12).

ward the same insulating state in which $\Delta \sim 600 \mathrm{~K}$. Nonetheless, the two types of disorder, (vanadium) vacancies versus substitution, must affect the states in the gap very differently for the variation of $\rho$ with $T \ll T_{M I}$ to be so dissimilar. Finally, we present in the inset to Fig. $2 \Delta$ as a function of total effective pressure, $P_{\text {tot }}$, for $\mathrm{V}_{1.9967} \mathrm{O}_{3}$. We define $P_{\text {tot }}$ as the sum of the external applied pressure and the internal chemical pressure due to the deviation from stoichiometry. ${ }^{13}$ The band gap diminishes linearly with increasing $P_{\text {tot }}$, extrapolating back to $\Delta=3500 \mathrm{~K}$ for pure $\mathrm{V}_{2} \mathrm{O}_{3}$, in agreement with previous measurements (solid circle). ${ }^{12}$ The observed linearity of $\Delta$ versus $P_{\text {tot }}$ indicates that the dopant and cation vacancies are uniformly distributed in the two samples.

We plot in Fig. $3 \ln \rho$ versus $(1 / T)^{1 / 4}$ for the Ti-doped sample at three pressures for $T$ well below $T_{M I}$. The solid lines are fits to three-dimensional variable range hopping, Eq. (1) with $\beta=\frac{1}{4}$. Although strong electronelectron interactions are essential to the physics of the Mott-Hubbard insulator, there are apparently still enough charge carriers available at these temperatures to screen the Coulomb gap. ${ }^{14}$ We find that the characteristic energy for the hopping state $T_{0}$ systematically decreases with increasing $P$ (inset to Fig. 3 ). It must go to zero at $P_{\text {tot }} \sim 25 \mathrm{kbar}$, where the metallic phase is stabilized at all $T$. We can estimate a localization length $\alpha^{-1}$ through the relation ${ }^{9,15} \alpha^{-1} \approx\left[k T_{0} N\left(E_{F}\right) / 16\right]^{1 / 3}$, where $N\left(E_{F}\right)$ is the density of states at the Fermi level and $k$ is Boltzmann's constant. Assuming an $N\left(E_{F}\right)$ of one state per vanadium atom, ${ }^{16}$ we find $\alpha^{-1}=0.6 \AA$ for $P=0$. Although $T_{0}$ is almost halved at $P=6 \mathrm{kbar}$, the localization length only increases by $20 \%$ because of the cube-root dependence.

We have analyzed the $\rho(T)$ dependence for $T<T_{M I}$ assuming purely electronic disorder. The contrast between nonstoichiometric and substitutionally-doped $\mathrm{V}_{2} \mathrm{O}_{3}$ so evident in Fig. 1 alternatively (or in addition) could reflect differences in the development of the antiferromagnetic order in the insulator. We compare, therefore, 


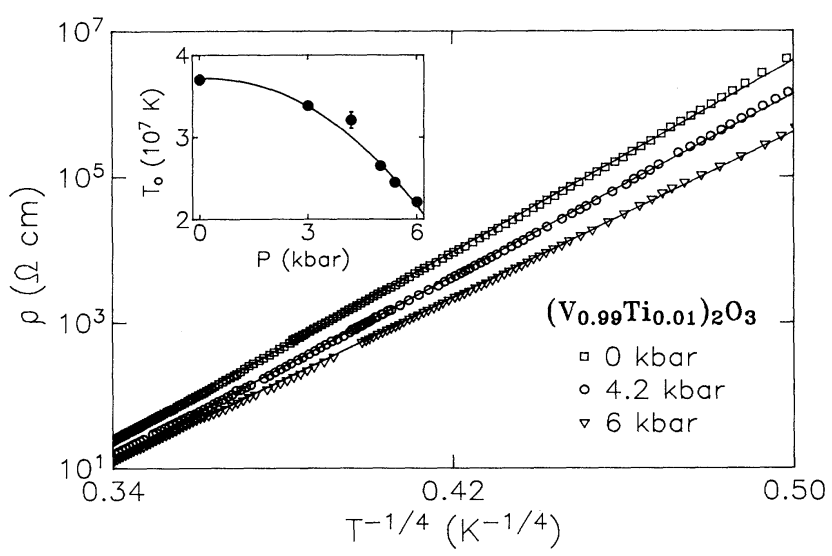

FIG. 3. Logarithm of the low-temperature resistivity $\rho$ vs $T^{-1 / 4}$ fit to a Mott variable range hopping form (solid lines). The inset shows the pressure dependence of the characteristic temperature $T_{0}$ [Eq. (1) in the text]. Line is a guide to the eye.

the temperature dependence at ambient pressure of the $c$ axis ac magnetic susceptibility $\chi$ for $\left(\mathrm{V}_{0.99} \mathrm{Ti}_{0.01}\right)_{2} \mathrm{O}_{3}$ and $\mathrm{V}_{1.9967} \mathrm{O}_{3}$ in Figs. 4(a) and 4(b), respectively. Both the magnitude and the general shape of $\chi(T)$ through the transition agree well with the original measurements. ${ }^{1,17}$ Moreover, the magnetic response appears to be indifferent to the nature of the disorder, indicating a similar role for the spin degrees of freedom in the two cases. Impurity states at the $1 \%$ level are not sufficient to affect profoundly the (short-range) superexchange spin-spin interaction. The arrows in Fig. 4 denote the appropriate thermal history. As observed in previous studies, ${ }^{1}$ we encounter a more pronounced hysteresis loop for the vanadium-deficient sample. We also find a slight upturn in the susceptibility at the lowest temperatures, an effect which becomes more important with increasing deviations from proper oxygen stoichiometry. ${ }^{13,17}$

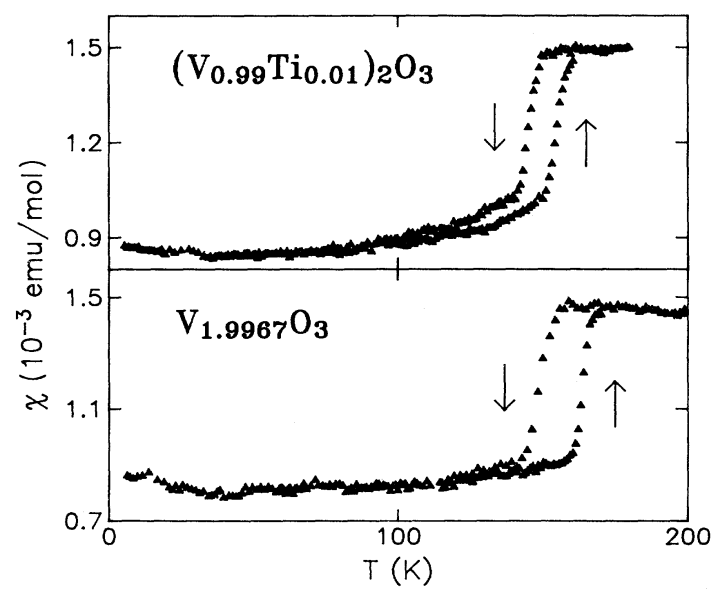

FIG. 4. The ac magnetic susceptibility $\chi$ as a function of temperature $T$ is essentially the same for substitutional and nonstoichiometric disorder. Down (up) arrows correspond to cooling (warming).
In the antiferromagnetic phase, the $\mathrm{V}_{2} \mathrm{O}_{3}$ spins lie at an angle of approximately $71^{\circ}$ away from the hexagonal $c$ axis. ${ }^{18}$ We compare in Fig. 5 the $a$ axis (upper) and $c$ axis (lower) magnetic susceptibilities for $\left(\mathrm{V}_{0.99} \mathrm{Ti}_{0.01}\right)_{2} \mathrm{O}_{3}$. We plot on the same figures the warming and cooling cycles for the $a$ axis and $c$-axis electrical resistivities over a similar temperature range. Other than roughly a $20 \%$ difference in the resistivity scale, ${ }^{19}$ the curves match, underlining the three-dimensional character of the system and the linkage of the resistive and magnetic instabilities. The drop in the magnetic response reflects the transition from a strongly enhanced Pauli susceptibility in the metal, due to the strong effective-mass enhancement of almost localized electrons with nearly atomic magnetic moments, to an ordered antiferromagnet in the insulator.

Our final comparison between substitutional and nonstoichiometric disorder involves calorimetry. We plot in Fig. 6 the heat capacity $C$ of the two samples in the range $0.3 \leq T \leq 5 \mathrm{~K}$. The solid lines are least-square fits to the form

$$
C=A / T^{2}+B T+D T^{3} .
$$

Here, the first term represents the Schottky tail from the vanadium hyperfine interaction, the second term corresponds to the possible presence of two-level systems due to disorder, and the third and final term accounts for both phonon and magnon contributions to the specific heat. We find a nuclear hyperfine splitting of 10.5 \pm 0.2 and $10.3 \pm 0.2 \mathrm{mK}$ for $\left(\mathrm{V}_{0.99} \mathrm{Ti}_{0.01}\right)_{2} \mathrm{O}_{3}$ and $\mathrm{V}_{1.9967} \mathrm{O}_{3}$, respectively. The experimental values concur, as they should for a local characteristic of vanadium itself, and they agree reasonably well with the previous determination $^{20}$ of $9.2 \mathrm{mK}$, which was measured in the range 0.1 to $0.5 \mathrm{~K}$.

In order to highlight the differences between the two types of disorder, we show in Fig. 7 the heat capacity less the fitted hyperfine contribution. In this plot of $C / T$ versus $T^{2}$, the slope represents the sum of the spin wave

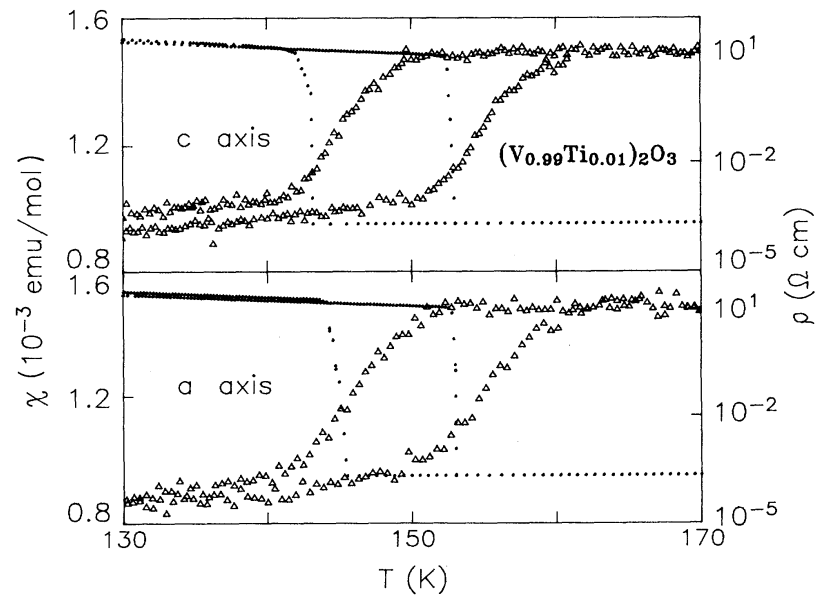

FIG. 5. Resistivity (dashed line, right axis) and susceptibility (open triangles, left axis) hysteresis loops through the metalantiferromagnetic-insulator transitions for both the $c$ and $a$ axes of $\left(\mathrm{V}_{0.99} \mathrm{Ti}_{0.01}\right)_{2} \mathrm{O}_{3}$. 


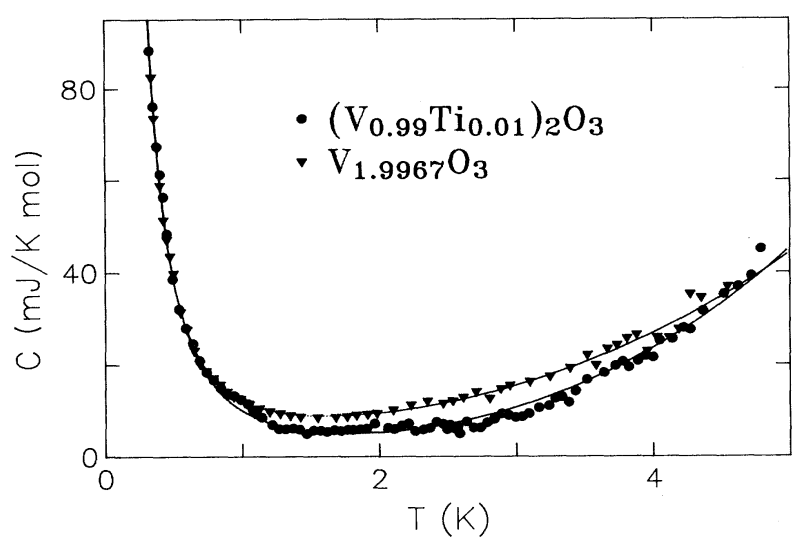

FIG. 6. Low-temperature specific heat $C$ vs temperature $T$ for both titanium-doped and vanadium-deficient $\mathrm{V}_{2} \mathrm{O}_{3}$. Upturn at low $T$ is due to the vanadium hyperfine contribution. Lines represent fits to Eq. (2) in the text.

and Debye lattice contributions and any nonzero intercept reflects the presence of two-level systems. The vanadium-deficient and substitutionally doped samples differ on both counts.

First, the slopes differ by almost $50 \%$, with the parameter $D$ in Eq. (2) equal to $0.25 \pm 0.02$ and $0.36 \pm 0.02$ $\mathrm{mJ} / \mathrm{K}^{4} \mathrm{~mol}$ for $\mathrm{V}_{1.9967} \mathrm{O}_{3}$ and $\left(\mathrm{V}_{0.99} \mathrm{Ti}_{0.01}\right)_{2} \mathrm{O}_{3}$, respectively. In both cases, we have normalized to one mole of $\mathrm{V}_{2} \mathrm{O}_{3}$. Given the similar behavior of $\chi$ for the two samples (Fig. 4), we posit that the difference in the prefactor to the $T^{3}$ term reflects a difference in the lattice, rather than the magnon, contribution to the heat capacity. The smaller slope for the vanadium-deficient sample corresponds to a larger Debye temperature relative to the $\mathrm{Ti}$ doped case. We can understand the difference in the Debye temperatures between the samples in terms of the different manner in which oxygen and titanium enter the lattice; the titanium enters substitutionally, while excess

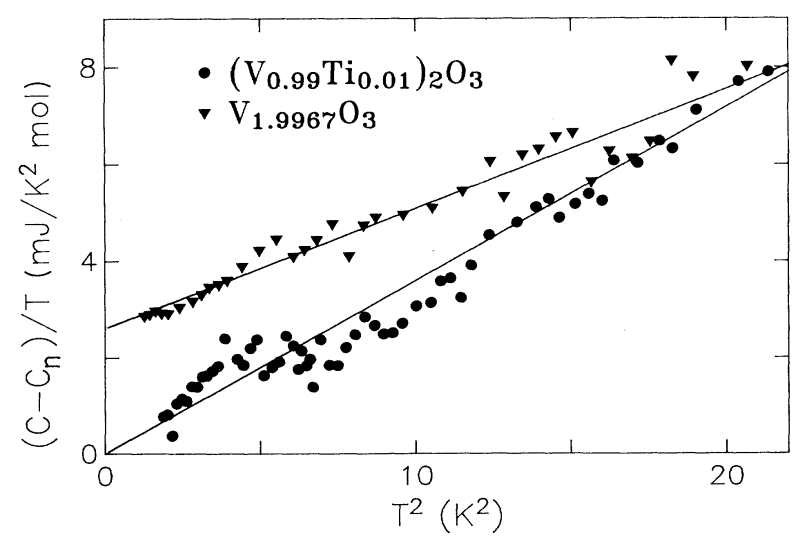

FIG. 7. Specific heat $C$ less the nuclear Schottky contribution, $C_{n} \propto 1 / T^{2}$. Lines represent least-square fits to a $T^{3}$ combined lattice and magnon term plus a linear $T$ term due to disorder. Only the vanadium-deficient sample shows evidence for a significant density of two-level systems. oxygen introduces vanadium vacancies. The larger $\mathrm{Ti}$ atom causes the crystal structure to realign itself such that the volume increases $0.1 \%$ per $1 \%$ titanium. ${ }^{21}$ By contrast, the introduction of vacancies into the lattice causes a crystal volume decrease of $0.1 \%$ per $0.5 \%$ vanadium deficiency. ${ }^{17}$ The decrease in volume implies that the nonstoichiometric sample has a more rigid lattice than the Ti-doped sample, in agreement with the heatcapacity measurements from which we deduce a greater Debye temperature for $\mathrm{V}_{1.9967} \mathrm{O}_{3}$ than for $\left(\mathrm{V}_{1.99} \mathrm{Ti}_{0.01}\right)_{2} \mathrm{O}_{3}$.

Second, only the specific-heat data for $\mathrm{V}_{1.9967} \mathrm{O}_{3}$ has a nonzero intercept. We obtain a value $B=2.6 \pm 0.2$ $\mathrm{mJ} / \mathrm{K}^{2} \mathrm{~mol}$ in that case, while this parameter is consistent with zero ( $\left.B=0 \pm 0.3 \mathrm{~mJ} / \mathrm{K}^{2} \mathrm{~mol}\right)$ for the substitutionally doped sample. The vanadium vacancies provide multiple sites for oxygen reorientations, which is the apparent source for the large density of two-level systems. In the standard model ${ }^{22}$ for the linear term due to disorder,

$$
C=2 n_{0} k^{2} T \int x^{2} \operatorname{sech}^{2} x d x,
$$

where $k$ is Boltzmann's constant and $n_{0}$ is the density of two-level systems, usually expressed in units of $\left(\mathrm{J}^{-1} \mathrm{~m}^{-3}\right)$. For $\mathrm{V}_{1.9967} \mathrm{O}_{3}, n_{0}=2.2 \times 10^{47} \mathrm{~J}^{-1} \mathrm{~m}^{-3}$. By comparison, ${ }^{23}$ a structural glass such as $\mathrm{SiO}_{2}$ (Spectrosil) has $n_{0}=8.4 \times 10^{45} \mathrm{~J}^{-1} \mathrm{~m}^{-3}$, while the Metglass $\mathrm{Zr}_{0.7} \mathrm{Pd}_{0.3}$ has $n_{0}=2.7 \times 10^{46} \mathrm{~J}^{-1} \mathrm{~m}^{3}$. The preponderance of two-level systems in vanadium-deficient $\mathrm{V}_{2} \mathrm{O}_{3}$ is manifested clearly in the low-temperature specific heat. These modes may also provide extra states in the gap which could account for the increased conductivity at low $T$ relative to substitutionally disordered $\mathrm{V}_{2} \mathrm{O}_{3}$ (Fig. 1).

\section{DISCUSSION AND INTERPRETATION OF RESULTS}

The low-temperature metal-insulator transition in $\mathrm{V}_{2} \mathrm{O}_{3}$ involves not only a discontinuous electronic transition, but accompanying magnetic and structural changes. Slater $^{24}$ first demonstrated that antiferromagnetic ordering can split a half-filled band, causing a transition from a paramagnetic metal to an antiferromagnetic insulator. However, experimental work by McWhan et al. ${ }^{25}$ showed that the Slater model could not account for the complete $\mathrm{V}_{2} \mathrm{O}_{3}$ phase diagram since the system undergoes a paramagnetic metal to paramagnetic insulator transition with chromium doping. Thus, the Mott-Hubbard ${ }^{1,26}$ model has been used to describe the metal-insulator transition in $\mathrm{V}_{2} \mathrm{O}_{3}$ because it is the simplest model which combines the effects of Coulomb and antiferromagnetic exchange interactions to force a band splitting.

In this section we will interpret our results within the approach of Spalek et al. ${ }^{3}$ which provides a rationalization of the full phase diagram for pure and doped $\mathrm{V}_{2} \mathrm{O}_{3}$ as a function of both temperature and doping. The generalized vanadium sesquioxide phase diagram obtained in Ref. 3 is schematically reproduced in slightly altered form in Fig. 8; the solid lines represent the first-order boundaries between the various phases: antiferromagnetic insulator (AFI), paramagnetic insulator (PI), and paramagnetic metal $\left(\mathbf{P M}\right.$ and $\left.\mathbf{P M}^{\prime}\right)$. The system is 
characterized by the ratio $U / U_{c}$, which represents the ratio of the intra-atomic Coulomb repulsion to the characteristic energy $U_{c}$, which is of order the bare bandwidth $W$. Three critical values of $U$ play a crucial role: (i) $U_{c} \sim W$; this parameter determines the upper stability limit of the PM phase with respect to the PI phase that would be achieved if no AF ordering intervened (as indicated by the dashed curve in Fig. 8); (ii) the parameter $U_{l c}$, which specifies the value of $U$ below which no PI phase can exist, and (iii) the parameter $U_{c}^{\prime}$, terminating the distinction between the reentrant metallic $\left(P M^{\prime}\right)$ and PI phases. As the temperature is raised, one encounters a sequence of phase changes whose nature is determined by the value of $U$, namely, (a) the $\mathrm{AFI} \rightarrow \mathrm{PM}$ transition only, for $U<U_{l c}$; (b) the $\mathrm{AFI} \rightarrow \mathrm{PM} \rightarrow \mathrm{PI} \rightarrow \mathrm{PM}^{\prime}$ sequence for $U_{l c}<U<U_{c}^{\prime}$; and (c) the $\mathrm{AFI} \rightarrow \mathrm{PI}$ transition only, for $U>U_{c}^{\prime}$. The first type (a) is encountered for pure $\mathrm{V}_{2} \mathrm{O}_{3}$, $\left(\mathrm{V}_{1-x} \mathrm{Ti}_{x}\right)_{2} \mathrm{O}_{3}$, and $\mathrm{V}_{2(1-y)} \mathrm{O}_{3}$; the second and third types occur in $\left(\mathrm{V}_{1-z} \mathrm{Cr}_{z}\right)_{2} \mathrm{O}_{3}$. All three cases are marked on Fig. 8. Note that pure $\mathrm{V}_{2} \mathrm{O}_{3}$ must be characterized by $U \approx U_{l c}$ since the PI and $\mathbf{P M}^{\prime}$ phases appear with small additions of $\mathrm{Cr} .^{12}$

We now concentrate on $\left(\mathrm{V}_{1-x} \mathrm{Ti}_{x}\right)_{2} \mathrm{O}_{3}$ and $\mathrm{V}_{2(1-y)} \mathrm{O}_{3}$. Even on the metallic side of the Mott-Hubbard transition, the electrons in the above compounds can be characterized as almost localized. Detailed calculations ${ }^{27}$ show that the antiferromagnetic insulating state below $U_{l c}$ can be of either the Slater or the Mott type. The Slater split-band picture is caused by the exchange field, the source of which is the antiferromagnetic exchange interaction. However, because of the relatively large mag-

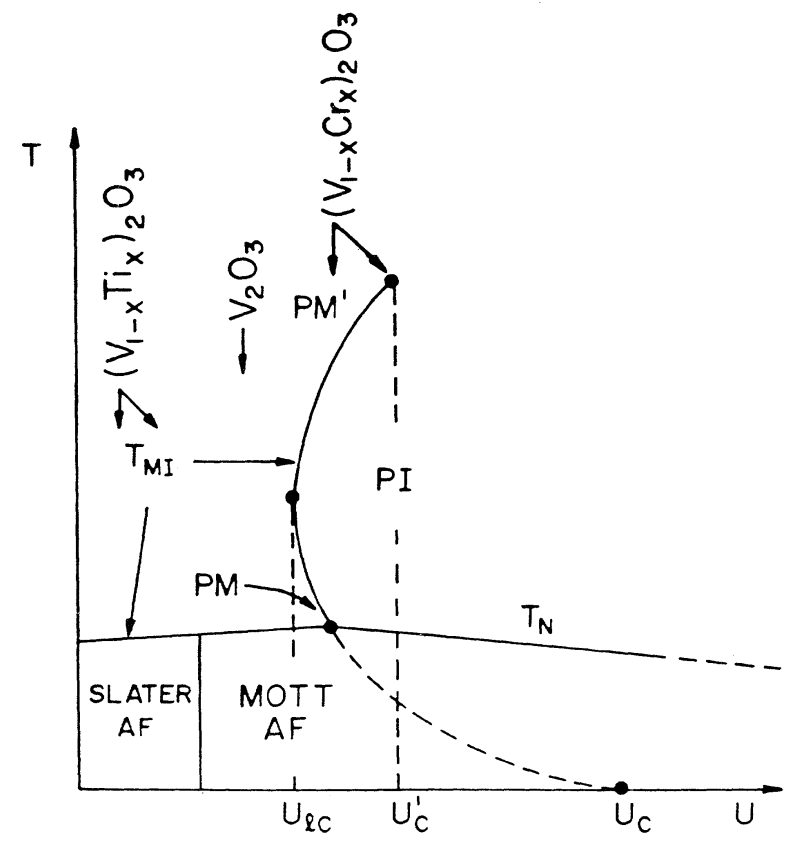

FIG. 8. Schematic representation of the phase diagram for the pure and doped $\mathrm{V}_{2} \mathrm{O}_{3}$ systems (adopted from Refs. 3 and 27). $U_{l c}$ characterizes the disappearance of the paramagnetic insulating phase sandwiched in between two metallic phases. nitude of $U$, the Slater subbands are substantially narrowed by electron correlation effects, as drawn schematically in Fig. 9. The band narrowing factor $\Phi$ may be estimated $^{27}$ as $\Phi \approx \Phi_{0}=1-\left(U / U_{c}\right)^{2}$; this estimate holds for a small sublattice magnetization. The effective Slater gap, $2 \bar{\Delta}$, is determined by the combined effect of the exchange splitting and the band narrowing which depends also on the amplitude of the sublattice moment $m_{\mathrm{AF}}$. If the magnetic moment in the ground state saturates ${ }^{28}$ (i.e., if $m_{\mathrm{AF}} \rightarrow 1$ ), then the lower Slater subband reduces to a discrete level split by combined Hubbard and exchange contributions, because $\Phi \rightarrow 0$ as $m_{\mathrm{AF}} \rightarrow 1$. Under these conditions, the system is a Mott insulator and the conduction gap is the (antiferromagnetic) Mott-Hubbard gap. ${ }^{29}$ The vertical transition line in Fig. 8 constitutes a first-order boundary between Mott and Slater antiferromagnet.

We have plotted in Fig. 2 the experimentally determined value for the conduction gap as a function of hydrostatic pressure for both the Ti-doped and vanadiumdeficient samples. In order to analyze the applicability of the Slater versus the Mott picture to the $\mathrm{V}_{2} \mathrm{O}_{3}$ systems studied here, we determine first the value of the gap due to the Slater contribution as

$$
\left.2 \bar{\Delta}=J_{\mathrm{AF}} z<S^{z}\right\rangle,
$$

where

$$
J_{\mathrm{AF}}=\frac{W^{2}}{z U}\left(1-\Phi_{0}\right)
$$

is the magnitude of the kinetic exchange interaction between $z$ nearest neighbors, applicable to the case with $U \leq U_{c} \cdot{ }^{3}$ If we regard $\mathrm{V}_{2} \mathrm{O}_{3}$ as an $S=1$ system, then the upper limit for the Slater gap is $2 \bar{\Delta}_{\max }=J_{\mathrm{AF}} z$. The paramagnetic Néel temperature $T_{N}^{\prime}$ is given by

$$
k T_{N}^{\prime}=\frac{1}{3} J_{\mathrm{AF}} z S(S+1)=\frac{2}{3} J_{\mathrm{AF}} z
$$

Therefore,

$$
2 \bar{\Delta}_{\max }=\frac{3}{2} k T_{N}^{\prime} .
$$

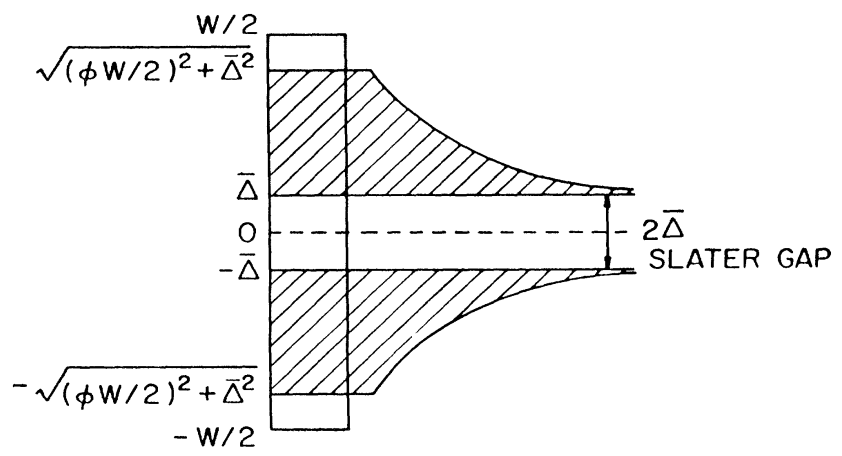

FIG. 9. The density-of-states (DOS) for quasiparticles (hatched parts) showing the Slater splitting with the gap $2 \bar{\Delta}$. The featureless form of the bare DOS is shown for comparison. $\Phi$ is the band-narrowing factor due to strong Coulomb interactions. 
With $T_{N}^{\prime} \approx 450 \mathrm{~K},{ }^{30} 2 \bar{\Delta}_{\max } \approx 675 \mathrm{~K}$, which is indeed the magnitude of the conduction gap we observe in the limit of high pressure (where the magnetic moment presumably has diminished). The larger value of $\Delta$ measured at low pressures in $\mathrm{V}_{1.9967} \mathrm{O}_{3}$ therefore must include as well a Mott-Hubbard contribution to the gap. Its decrease with added pressure and subsequent approach to the Slater limit is expected within the Mott-Hubbard model since pressure increases the bandwidth $W$ and, in a simple approximation, $\Delta \sim U-W$. For the case of $\left(\mathrm{V}_{1-x} \mathrm{Ti}_{x}\right)_{2} \mathrm{O}_{3}$, the magnetic moment diminishes rapidly with $\mathrm{Ti}$ content ${ }^{31}$ and the crossover to the Slater dominated regime must occur at much lower pressures.

It is important to note that although the $\mathrm{V}_{2} \mathrm{O}_{3}$ samples studied here may verge on being Slater antiferromagnets, they are still on the Mott side of the boundary for the highest pressures applied since the proper value of $2 \bar{\Delta}_{\max } \sim \Delta$ is obtained only when the magnetic moment is assumed to remain saturated in the insulating state. We remark also that the effect of disorder introduced by $\mathrm{Ti}$ or oxygen vacancies on the observed gap in the AFI phase is not likely to be a predominant factor since (unlike the Anderson transition) the PM $\rightarrow$ AFI transformation is of first order and accompanied by the onset of AF ordering.

We cannot overlook one additional complication. The $\mathrm{Ti}^{3+}$ cation possesses only one $3 d$ electron while $\mathrm{V}^{3+}$ has two. Hence, $\mathrm{Ti}^{3+}$ ions introduce holes in either the $a_{1}$ or the $e_{\pi}$ band. The observed weak anisotropy of the transport properties can be reconciled with the strongly anisotropic character of the $e_{\pi}$ and $a_{1}$ orbitals $^{32}$ by noting that there should be appreciable hybridization of these two types of $3 d$ states with the oxygen $3 s$ and $3 p$ states (the hybridization with the $2 p$ states is small since they are located roughly $4 \mathrm{eV}$ below the Fermi level ${ }^{33}$ ). Thus, the observed $\mathrm{Ti}^{3+}$ hole localization must be ascribed to a local distortion of the antiferromagnetic ordering via spin polarons (cf. Mott ${ }^{1}$ ) or via spin-bag effects explored recently in the context of hole doping in high- $T_{c}$ systems. ${ }^{34}$ The binding of the hole locally distorts the average Slater or Mott-Hubbard gap and creates an insulating state even in the slightly-less-than-half-filled band situation.

We now try to understand the observed differences in the low temperature $\rho(T)$ of the two systems. The $\mathrm{Ti}^{3+}$ holes simply participate in the hopping conduction, which leads to the $\exp \left(T_{0} / T\right)^{1 / 4}$ dependence of the resistivity plotted in Fig. 3. In the vanadium-deficient system, the $\mathrm{V}$ vacancy removes an electron from both the $e_{\pi}$ and $a_{1}$ states. The conduction mechanism appears to be strongly influenced by the added states in the gap due to disorder, leading to an increased conductivity at low $T$. It would be interesting to study the hopping conduction in $\left(\mathrm{V}_{1-z} \mathrm{Cr}_{z}\right)_{2} \mathrm{O}_{3}$ for which $U / U_{c}$ is increased and, therefore, is driven towards the limit of strongly correlated electrons.

One should inquire why with such different conduction processes in the insulating phase, the pressure dependence of $T_{M I}$ remains the same in the two systems. We note first that the number of electrons participating in the conduction process is small, i.e., the carrier concentration varies as $\sim x$ or $\sim 2 y$ in the two cases, respectively. By contrast, the stability of the AFI phase relative to the PM phase is determined by the global energy balance involving all $d$ electrons. To prove this point in a quantitative fashion, we use the theoretical model introduced earlier ${ }^{3}$ to calculate the phase diagram for the $\left(\mathrm{V}_{1-z} \mathrm{Cr}_{z}\right)_{2} \mathrm{O}_{3}$ system. In this model, the free energy of the PM state in the low-temperature regime is given by

$$
\frac{F_{M}(T)}{M}=\bar{\varepsilon}\left(1-\frac{U}{U_{c}}\right)^{2}-\frac{1}{2} \frac{\gamma_{0}}{\Phi_{0}} T^{2},
$$

where $\bar{\varepsilon}$ is the bare band energy per atom, $U_{c}=8|\bar{\varepsilon}| \approx 2 \mathrm{~W}$ is the upper critical value of $U$ for the Mott-Hubbard transition at $T=0$, and $\gamma_{0}=\frac{2}{3} \pi^{2} k^{2} \rho\left(\varepsilon_{F}\right)$ is the linear specific-heat coefficient (per site) for the electrons. By contrast, the free energy of the Mott insulating phase can be estimated as

$$
F_{\mathrm{AF}}=-J_{\mathrm{AF}} z / 8+A T^{4}
$$

where the second term is the thermal contribution due to long-wavelength acoustic magnon excitations and $A$ is related to the exchange-stiffness constant.

The first-order AFI $\rightarrow$ PM transition temperature $T_{M I}$ is determined by the coexistence condition $F_{\mathrm{AF}}=F_{M}$, which yields

$T_{M I}=\frac{1}{2 A}\left\{\left[\left[\frac{\gamma_{0}}{2 \Phi_{0}}\right]^{2}+4 A \Delta E\right]^{1 / 2}-\frac{1}{2}\left[\frac{\gamma_{0}}{\Phi_{0}}\right]\right\}^{1 / 2}$,

where

$$
\Delta E=\bar{\varepsilon}\left(1-\frac{U}{U_{c}}\right)^{2}+J_{\mathrm{AF}} z / 8
$$

is the difference between the energies in the metallic and insulating states. Positive values of $T_{M I}$ occur only for $\Delta E>0$, that is, when the stable phase at $T=0$ is antiferromagnetic. As $U \rightarrow U_{c}, \Delta E$ increases $(\bar{\varepsilon}<0)$, and so does $T_{\text {MI }}$. On the other hand, on applying either an external or a chemical pressure one increases $W$ (i.e., $\bar{\varepsilon}$ ) as well as $U_{c}$, which reduces $\Delta E$ towards zero. Hence, $T_{M I}$ should decrease similarly with pressure for both $\left(\mathrm{V}_{1-x} \mathrm{Ti}_{x}\right)_{2} \mathrm{O}_{3}$ and $\mathrm{V}_{2(1-y)} \mathrm{O}_{3}$ if we make the reasonable assumption that one vanadium vacancy removes two electrons from the $3 d$ band. This is indeed observed (Fig. 1). A more quantitative estimate of the variation of $T_{M I}$ with $P$ cannot be provided since Eq. (6) contains parameters such as $W, U$, and $A$, the accuracy of which cannot be estimated reliably at present.

We note one final important feature of the first-order AFI $\rightarrow$ PM transition, namely, that $T_{M I}$ is substantially lower than either the effective Fermi energy $\Phi_{0} \varepsilon_{F} / k$ (where $\varepsilon_{F}$ is the bare Fermi energy) or the intrinsic Néel temperature $T_{N}^{\prime}$. This is why we have adopted the lowtemperature expression for both $F_{M}$ and $F_{A F}$.

\section{CONCLUSIONS}

The first-order metal-insulator transition temperature is identically reduced with hydrostatic pressure for 
$\left(\mathrm{V}_{0.99} \mathrm{Ti}_{0.01}\right)_{2} \mathrm{O}_{3}$ and $\mathrm{V}_{1.9967} \mathrm{O}_{3}$, i.e., in samples for which $y \approx x / 2$. However, the nature of the insulating state differs, as characterized by both the magnitude and the pressure dependence of the gap as well as the form of the low-temperature transport $\left(T<<T_{M I}\right)$. Low-temperature calorimetry reveals the presence of significant low-energy excitations due to disorder in only the vanadium-deficient samples. By contrast, magnetic susceptibility measurements indicate that the spin degrees of freedom are insensitive to the particular form of disorder. We qualitatively explain these experimental parallels and differences in terms of a single narrow-band model for $3 d$ electrons with the ratio $U / U_{c} \sim U / W$ as a microscopic parameter characterizing a given compound. Our results point to a crossover from Mott insulator to Slater insulator with increasing pressure.

\section{ACKNOWLEDGMENTS}

The work at The University of Chicago was supported by National Science Foundation (NSF) Grant No. DMR 8816817. J. Yang acknowledges support from NSF Grant No. DMR 8351992. The Purdue effort was supported by NSF Grant No. DMR 8616533. J. Spalek acknowledges the support of the Superconductivity Center at Purdue. We are most grateful to Patricia Metcalf for reannealing the crystals used in this research.
${ }^{1}$ For reviews, see D. B. McWhan, A. Menth, and J. P. Remeika, J. Phys. (Paris) Colloq. 32, C1-1079 (1971); S. A. Shivashankar and J. M. Honig, Phys. Rev. B 28, 5695 (1983); N. F. Mott, Metal-Insulator Transitions (Taylor and Francis, London, 1974).

${ }^{2}$ W. F. Brinkman and T. M. Rice, Phys. Rev. B 2, 4302 (1970).

${ }^{3}$ J. Spalek, A. Datta, and J. M. Honig, Phys. Rev. Lett. 59, 728 (1987).

${ }^{4}$ J. C. C. Fan and T. B. Reed, Mater. Res. Bull. 7, 1403 (1972).

${ }^{5}$ J. E. Keem, H. R. Harrison, S. P. Faile, H. Sato, and J. M. Honig, Am. Ceram. Soc. Bull. 56, 1022 (1977); H. R. Harrison, R. Aragon, and C. J. Sandberg, Mater. Res. Bull. 15, 571 (1980).

${ }^{6}$ S. A. Shivashankar, R. Aragon, H. R. Harrison, C. J. Sandberg, and J. M. Honig, J. Electrochem. Soc. 128, 2472 (1981); 129, 1641(E) (1982).

${ }^{7}$ M. Wun and N. E. Phillips, Cryogenics 15, 36 (1975).

${ }^{8}$ D. B. McWhan, A. Menth, J. P. Remeika, W. F. Brinkman, and T. M. Rice, Phys. Rev. B 7, 1920 (1973).

${ }^{9}$ N. F. Mott, Philos. Mag. 19, 635 (1969); N. F. Mott and E. A. Davis, Electronic Processes in Non-Crystalline Materials, 2nd ed. (Oxford University Press, London, 1979).

${ }^{10}$ A. L. Efros and B. I. Shklovskii, J. Phys. C 8, L49 (1975).

${ }^{11}$ N. F. Mott, Philos. Mag. 24, 911 (1971).

${ }^{12}$ H. Kuwamoto, J. M. Honig, and J. Appel, Phys. Rev. B 22, 2626 (1980).

${ }^{13}$ D. B. McWhan, A. Menth, and J. P. Remeika, J. Phys. (Paris) Colloq. 32, C1-11, (1971).

${ }^{14}$ See, for comparison, crossover behavior from $\beta=\frac{1}{2}$ to $\beta=\frac{1}{4}$ in $\mathrm{La}_{2-x} \mathrm{Sr}_{x} \mathrm{CuO}_{4}$ : B. Ellman, H. M. Jaeger, D. P. Katz, T. F. Rosenbaum, A. S. Cooper, and G. P. Espinosa, Phys. Rev. B 39, 9012 (1989).

${ }^{15}$ V. Ambegaokar, B. I. Halperin, and J. S. Langer, Phys. Rev. B 4, 2612 (1972).

${ }^{16}$ C. Castellani, C. R. Natoli, and J. Ranninger, J. Phys. (Paris) Colloq. 37, C4-199 (1976).
${ }^{17}$ Y. Ueda, K. Kosuge, and S. Kachi, J. Solid State Chem. 31, 171 (1980).

${ }^{18}$ R. M. Moon, Phys. Rev. Lett. 25, 527 (1970); J. Appl. Phys. 41, 883 (1970).

${ }^{19}$ S. A. Shivashankar, Ph.D. thesis, Purdue University, 1981 (unpublished).

${ }^{20}$ K. Andres, Phys. Rev. B 2, 3768 (1970).

${ }^{21}$ S. Chen, J. E. Hahn, C. E. Rice, and W. R. Robinson, J. Solid State Chem. 44, 192 (1982).

${ }^{22}$ P. W. Anderson, B. I. Halperin, and C. M. Varma, Philos. Mag. 25, 1 (1972); W. A. Phillips, J. Low Temp. Phys. 7, 351 (1972).

${ }^{23}$ R. O. Pohl, in Amorphous Solids: Low Temperature Properties, edited by W. A. Phillips (Springer-Verlag, Berlin, 1981), p. 27.

24J. C. Slater, Phys. Rev. 82, 538 (1951).

${ }^{25}$ D. B. McWhan, T. M. Rice, and J. P. Remeika, Phys. Rev. Lett. 23, 1384 (1969).

${ }^{26}$ J. Hubbard, Proc. R. Soc. London Ser. A 281, 401 (1964).

${ }^{27}$ J. Spalek (unpublished). For a review, see J. M. Honig and J. Spalek, Proc. Indian Sci. Acad. 52A, 232 (1986); A. Datta, Ph.D. thesis, Purdue University, 1988 (unpublished).

${ }^{28} \mathrm{We}$ disregard any moment reduction caused by the zero-point motion in the Néel state of an antiferromagnet.

${ }^{29}$ In reality, the magnetic moment is $\mu=1.2 \mu_{B}$ per $V^{3+}$ ion (Ref. 18), which is considerably below the expected saturation value $2 \mu_{B}$.

${ }^{30}$ A. Menth and J. P. Remeika, Phys. Rev. B 2, 3756 (1970).

${ }^{31}$ Y. Ueda, K. Kosuge, S. Kachi, and T. Takada, J. Phys. (Paris) Colloq. 40, C2-275 (1979).

32J. Ashkenazi and M. Weger, Adv. Phys. 22, 207 (1973); J. Phys. (Paris) Colloq. 37, C4-189 (1976).

${ }^{33}$ K. E. Smith and V. E. Heinrich, Phys. Rev. B 38, 5965 (1988).

${ }^{34}$ J. R. Schrieffer, X. G. Wen, and S. C. Zhang, Phys. Rev. B 39, 11663 (1989). 